\title{
A Comparison of Direct and Indirect Multi-touch Input for Large Surfaces
}

\author{
Dominik Schmidt, Florian Block, and Hans Gellersen \\ Computing Department, Lancaster University, Lancaster, UK \\ \{schmidtd, block, hwg\}@ comp. lancs.ac.uk
}

\begin{abstract}
Multi-touch input on interactive surfaces has matured as a device for bimanual interaction and invoked widespread research interest. We contribute empirical work on direct versus indirect use multi-touch input, comparing direct input on a tabletop display with an indirect condition where the table is used as input surface to a separate, vertically arranged display surface. Users perform significantly better in the direct condition; however our experiments show that this is primarily the case for pointing with comparatively little difference for dragging tasks. We observe that an indirect input arrangement impacts strongly on the users' fluidity and comfort of 'hovering' movement over the surface, and suggest investigation of techniques that allow users to rest their hands on the surface as default position for interaction.
\end{abstract}

Keywords: Multi-touch interfaces, surface computing, indirect input.

\section{Introduction}

Multi-touch input on interactive surfaces has been studied for over 25 years, from early work on tablets as separate input device [14] to more recent direct multi- touch on interactive tabletops [7, 10]. Multi-touch supports natural use of both hands for bimanual tasks and greatly expands the range of gestural input that can be used in interaction $[6,26]$. This is inspiring widespread research activity on multi-touch interactive tabletops [22], but empirical insight into multi-touch input performance is still limited.

Recent work on multi-touch input has tended to imply its use for direct interaction. As noted by Forlines et al. [9], it is commonly argued that direct touch is more "natural" or "compelling" than working with an indirect input de- vice. Forlines et al. took this to motivate a study comparing direct multi-touch with indirect mouse input (providing evidence for their respective advantages for bimanual and single-pointer tasks). We, in turn, ask how much the benefit of multi-touch for bimanual tasks depends on the directness of the input. Intuitively, direct multi-touch would appear superior to indirect multi-touch. However indirect interaction, where the hands are off the output medium, can have a variety of advantages depending on application context, for example interaction at-a-distance, separate input surfaces for multiple users, avoidance of occlusions [21], or one input surface to multiple displays [19].

The contribution of this paper is an empirical study of direct versus indirect use multi-touch input. The study compares direct input on a tabletop display with an 
indirect condition where the table is used for input only, with output on separate display. Figure 1 illustrates the two conditions. In the direct condition, input and output space coincide. In the indirect condition, output is on a display arranged vertically behind the input surface; for reference between input and output space, the user's hand contours are displayed.

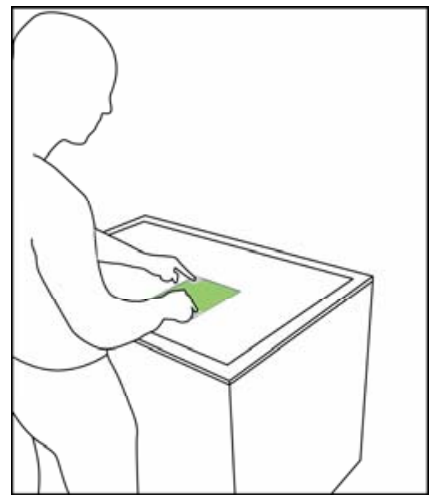

(a) Direct multi-touch: input and output space coincide

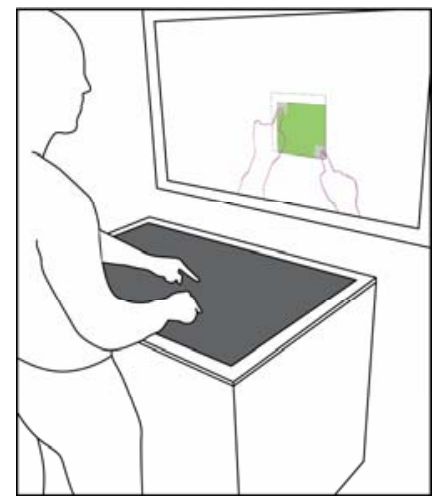

(b) Indirect multi-touch: separate output with display of hand contours

Fig. 1. Direct versus indirect multi-touch interaction

We describe an experiment that investigates direct versus indirect multi-touch for a symmetric bimanual task, in terms of quantitative performance, qualitative observations, and user preference. The results overall indicate that users are faster with multitouch for direct interaction but are also able to use multi-touch efficiently for indirect input. A main observation was that users approach tasks differently in the two conditions, in the direct condition with fluid movement to a target, and in the indirect condition with hovering movement until a target has reached, resulting not only in longer selection time but also less comfort. The discomfort with hovering over the surface to avoid accidental touch suggests adoption of multi-touch techniques that would allow user to rest their hands on the surface for interaction.

\section{Related Work}

The performance of direct and indirect input modalities for bimanual tasks on interactive surfaces has been scarcely explored. Forlines et al. compared direct- touch with mouse input for bimanual and single-pointer tasks, highlighting their different advantages [9]. Barnert described a similar experiment on dual-mouse versus multi-touch [2]. These studies provide some insight into suitability for the respective interfaces for unimanual versus bimanual tasks. Our work, in contrast, is focused on a single input modality (multi-touch on interactive surfaces), to analyze its use and performance in direct versus indirect interaction. 
Direct versus indirect input has been studied widely for single-pointer interaction, comparing indirect input devices with direct-touch or pen input (e.g., [20, 23]). A recent study is close to ours in spirit, as it focuses on a single in- put technology, here a stylus pen, which is compared for direct and indirect interaction showing that indirect use can perform as well for certain tasks [8].

Multi-touch technology has become closely associated with interactive displays and direct interaction, however a variety of recent systems have been based on multitouch input separate from output. Malik et al. demonstrated a system that integrates multi-touch input for interaction with multiple displays in the environment [19]. In other work, Moscovich et al. investigate indirect multi- touch techniques aimed to overcome limitations of direct-touch interaction on touchscreens (e.g., limited precision, occlusion issues, and limitations on size and proximity of the display) [21].

In this work we focus on the use of multi-touch technology that has more recently evolved with interactive tabletops $[10,26]$. However, our work also ties in with a large body of earlier work on bimanual interaction (e.g., $[1,5,13,15])$.

To support indirect multi-touch, we track and display hand contours. This concept was first demonstrated in Krueger et al.'s Videoplace [12]. In recent work, a related technique was used for 'see-through' multi-touch input on the back of a mobile device, addressing occlusion and precision limitations of multi-touch 'on the front' of the device [24].

\section{Experimental Design}

The principal aim of our study was to gain insight into direct versus indirect use of multi-touch input for bimanual tasks. We specifically sought to understand performance differences (are users slower when input is indirect, and how much so?), principal obstacles in using multi-touch indirectly (are users able to use multi-touch effectively when input is displaced from output?), and potential qualitative differences in interaction behavior (do users approach tasks differently when input is separate from output?).

22 users took part in the study (10 female, 12 male). They were recruited from the local campus and compensated with $£ 8$ for their time. The age ranged from 18 to 53 years with an average of 27.23 years $(\mathrm{SD}=7.79)$. All participants but one were righthanded and regular computer users without specific experience in bimanual interfaces.

\subsection{Apparatus}

The experiment was designed to compare direct and indirect multi-touch in the configuration we introduced in Figure 1. Both conditions use the same tabletop technology as input surface. In the direct condition, the surface doubles as display. In the indirect condition, the display is provided on a separate screen arranged vertically behind the input surface. In both conditions, the respective other display is turned off. The size of the vertical display is chosen to appear, from the user's perspective, to be the same size as the input surface. This preserves the 1:1 ratio of input and output spaces across the two conditions. Mapping between input and output is supported with a contour display of the user's hands, and with display of circles as feedback for points of touch. 


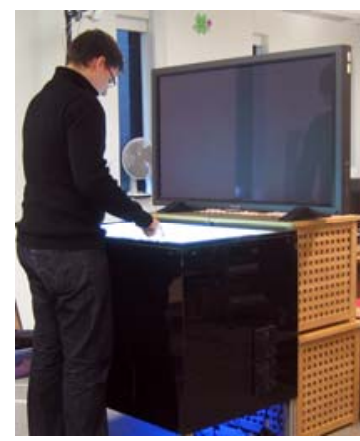

(a) Direct multi-touch

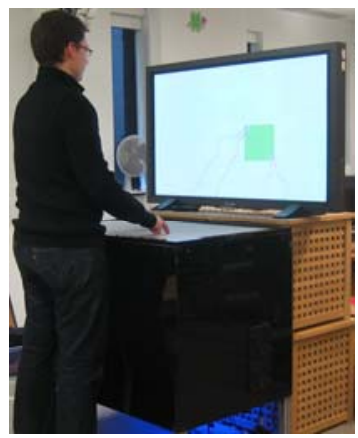

(b) Indirect multi-touch

Fig. 2. User interacting with multi-touch table and vertical screen

The experimental setup is shown in Figure 2. The tabletop had a surface diagonal of $100 \mathrm{~cm}$ with rear projection display of $1280 \times 768 \mathrm{px}$. Touch detection was based on a diffused illumination principle, using a Point Grey Firefly MV camera with a resolution of $640 \times 480 \mathrm{px}$ at $60 \mathrm{fps}$ to capture points of touch. The vertical display was provided by a $126 \mathrm{~cm}$ plasma screen with the same resolution as the tabletop display. A second camera (same model) was mounted on the ceiling to track the position and contour of the user's hands over the tabletop. The hands were extracted from the captured image, processed by an edge detection algorithm, and mapped using homography.

\subsection{Task}

Figure 3 illustrates the 2D symmetric bimanual task used in this experiment. In order to build upon the literature, the task was designed according to the bimanual task in

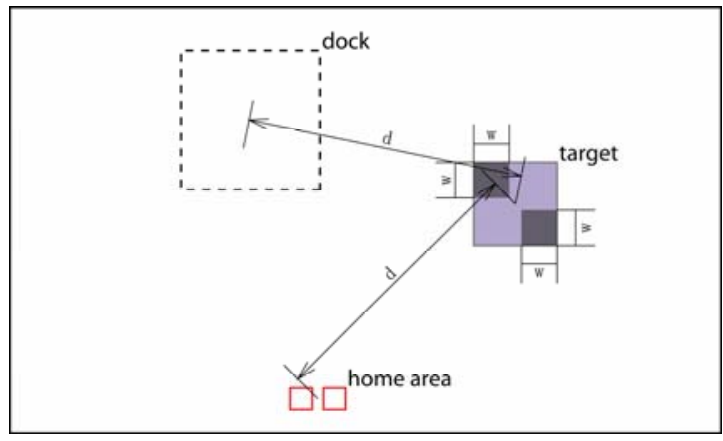

(a) Task layout: home area, target, and dock; same distance $\mathrm{d}$ between home area and target respectively target and dock; square handles of side length $\mathrm{w}$

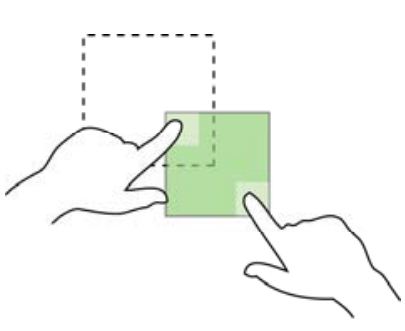

(b) Dragging and resizing selected target to match dock

Fig. 3. Task design: pointing (starting in home area) and dragging (target onto dock) 
Forlines et al.'s [9] experiment. It consists of a pointing and a dragging subtask, both common interaction techniques in direct manipulation interfaces, but shown to yield difference performance behavior [18]. Additionally, the dragging subtask requires the user to simultaneously perform a resizing operation similar to the "stretchies" technique proposed by Kurtenbach et al. [13] but limited to translation and scaling.

At the beginning of each task, a home area is shown at an arbitrary location, consisting of two red, non-filled squares. Participants were instructed to touch these squares, using one finger of each hand. To prevent participants from rushing through tasks, a waiting time was introduced before target and dock appeared. To complete the pointing subtask, participants had to select the target's two handles. The target was rendered as blue, semi-transparent square with two square handles in the opposite corners. While the target had a fixed size of 150px square the handles' sizes were varied as part of the experiment. Providing visual feedback, selected handles were highlighted in a brighter color. Moreover, once both handles - and hence the targetwere selected the target's color changed from blue to green.

To complete the dragging subtask, participants had to match the target with the dock by translating and scaling the target (compare figure 3(b)). The dock was rendered as outlined square with a dashed, black border at a fixed size of 250px square. The target's size could be adjusted by changing the handle's relative position. It was sufficient to match the four edges accurately within $10 \mathrm{px}$ each which was indicated by changing the dock's border and fill color. The dragging task was successfully completed once target and dock were matched and the user had released at least one of the handles.

Home area, target, and dock positions were randomized but precalculated for all trials, i.e. each user was presented with the same position constellations. In addition to the handle size, the distance between home area and target respectively target and dock was simultaneously varied as part of the experiment. Participants were instructed to perform the task as quickly and accurately as possible. It was not possible to move on to the next task before the matching and hence the task was completed successfully.

\subsection{Design and Procedure}

A repeated-measures design was used with the within participant independent variables interaction condition (direct, indirect), handle width $(32,48,64 \mathrm{px})$, and distance (300, 500, 700px). Every combination of handle width and distance was repeated three times. With 27 trials per block, every user performed five blocks resulting in 135 trials per user and condition and a total number of 5940 trials.

Participants were introduced to the interface and the concept of multi-touch interaction. The experiment started with two training blocks which were followed by the three blocks used in the analysis. Participants completed both input conditions in succession and their order was counterbalanced. Each participant was presented with the same set of precalculated trials. However, the presentation order of trials within a block was pseudo-randomized for every user. The same set of blocks was used for testing the direct and indirect conditions.

After each condition, participants were asked to state their agreement with eight items ${ }^{1}$ selected from the IBM Computer Usability Satisfaction Questionnaire [16] on

\footnotetext{
${ }^{1}$ Statements 2, 4, 5, 6, 7, 9, 17, and 19 of the Post-Study System Usability Questionnaire were selected as applicable to the test system.
} 
a seven point Likert scale ranging from "strongly agree" to "strongly disagree". In addition, participants were also asked to rate the amount of mental demand required to fulfill the task as well as their frustration level and to give a self-assessment of their performance, using three items from the NASA Task Load Index [11]. In the end of the experiment, participants were asked to state a preference and their subjective rating about performance and error rates for each condition.

The measured variables were overall trial time (pointing + dragging time), pointing time, dragging time, pointing errors, dragging errors, and handle selection delay. Additionally, we recorded video footage of all participants with the camera placed at the shorter side of the table slightly above its surface to capture arm and hand movements as well as hand postures.

\section{Results}

\subsection{Quantitative Measurements}

Trial Time. Trial time is the sum of pointing and dragging time. It was measured in milliseconds from the moment when the user left the home area to the moment when he or she completed the docking successfully. A 3-Way Repeated Measures ANOVA of the recorded data indicates a significant main effect of condition on the trial time, $F_{1,21}=216.86, p<0.001$. Participants completed a trial quicker in the direct condition $(M=4311, S D=1787)$ than in the indirect condition $(M=6700, S D=2704)$. Figure 4(a) shows mean trial times broken down by pointing and dragging time.

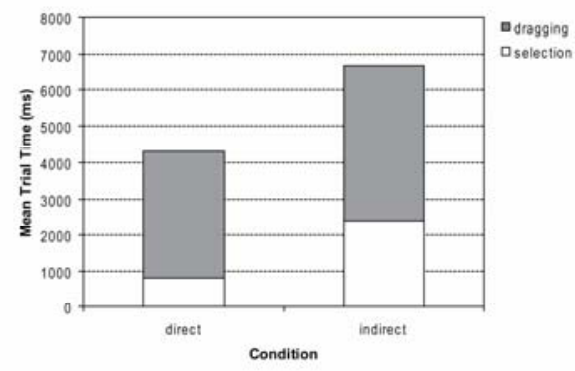

(a) Mean trial time for each condition broken down by pointing and dragging time

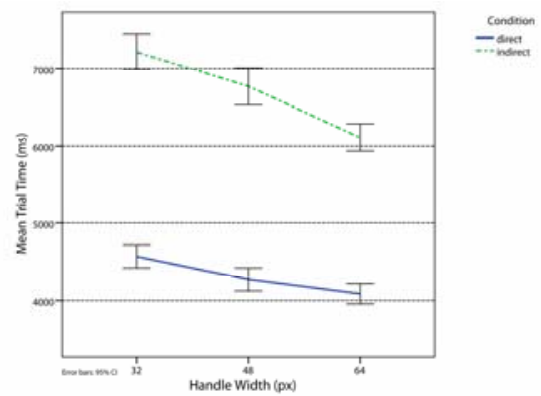

(b) Mean trial time for each condition broken down by width

Fig. 4. Mean trial times

Both, width and distance have main effects on trial time, $F_{2,42}=45.05, p<0.001$ and $F_{2,42}=91.94, p<0.001$ for width and distance respectively. The only significant interaction revealed by the analysis is condition $\times$ width, $F_{2,42}=5.99, p=0.005$, indicating that the effect of width on trial time differed for the two conditions. Contrasts on this interaction term revealed that when the difference in trial time between direct and indirect condition was compared for 32 and 48px there was no significant 
difference, $F_{2,42}=0.56, p=0.465$. However, comparing the difference in trial time between direct and indirect for 48 and 64px, a significant difference emerged $\left(F_{2,42}=7.75, p=0.011\right)$ which indicates that the decline in trial time between 48 and $64 \mathrm{px}$ was significantly more pronounced in the indirect condition (compare figure 4).

Pointing Time. Pointing time was measured in milliseconds from the moment when the user left the home area to the moment when he or she had success- fully acquired both of the target's handles. As erroneous trials were not repeated the user continued until the selection was successful. Therefore, pointing time includes time required to correct errors. An ANOVA of the recorded data indicates that pointing time was significantly shorter in the direct $(M=811, S D=505)$ than in the indirect condition $(M=2386, S D=995), F_{1,21}=357.75, p<0.001$.

Both, width and distance, have main effects on pointing time, $F_{1.63,34.14}=162.26$, $p<0.001$ (corrected degrees of freedom using Huynh-Feldt estimates of sphericity) and $F_{2,42}=91.09, p<0.001$ for width and distance respectively. The condition $\times$ width interaction is significant, $F_{1.6,33.61}=37.86, p<0.001$ (Huynh- Feldt), and so is the condition $\times$ distance interaction $\left(F_{2,42}=19.06, \mathrm{p}<0.001\right)$, indicating that the effect of width respectively distance on pointing time differed for the two conditions. No significant interaction between condition, width, and distance was found. Figure 5 shows the mean pointing time for each width and distance, separated for each condition.

Contrasts on the condition $\times$ width interaction term revealed that when the difference in pointing time between direct and indirect condition was compared for 32 and 48px respectively 48 and 64px, significant differences emerged in both comparisons $\left(F_{1,21}=27.7, \mathrm{p}<0.001\right.$ respectively $\left.F_{1,21}=19.73, \mathrm{p}<0.001\right)$. Likewise, contrasts indicated a significant differences in pointing time between direct and indirect condition for 300 and 500px $\left(F_{1,21}=5.47, \mathrm{p}=0.029\right)$ and 500 and $700 \mathrm{px}\left(F_{1,21}=23.21\right.$, $\mathrm{p}<0.001)$. To sum up, the decline in pointing time for larger handles as well as shorter distances was significantly more pronounced in the indirect condition.

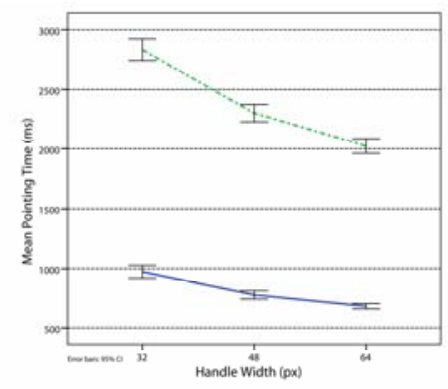

(a) Broken down by width
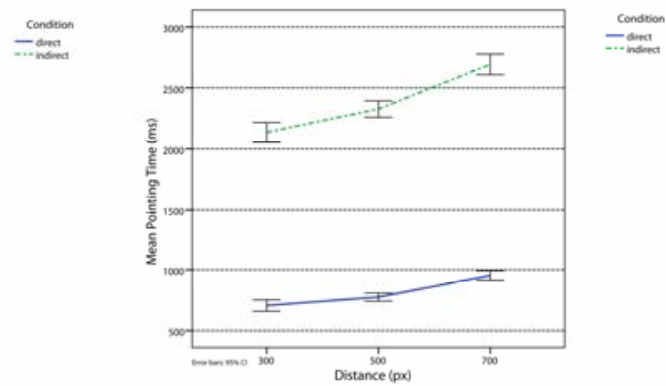

(b) Broken down by distance

Fig. 5. Mean pointing time for each condition

Pointing Errors. A pointing error would be registered if the participant missed either handle. Multiple pointing errors could occur as the trial would not be reset if an error was made. An ANOVA shows that significantly fewer errors were made in the direct 
$(M=0.16, S D=0.6)$ than in the indirect condition $(M=0.57, S D=1.21)$, $F_{1,21}=55.48, p<0.001$.

Both, width and distance, have main effects on pointing error, $F_{2,42}=14.66$, $p<0.001$ and $F_{2,42}=6.9, p=0.003$ for width and distance respectively. No significant interaction between condition and width or distance was found.

Dragging Time. Dragging time was measured in milliseconds from the moment of successful acquisition of both handles to the moment the user released either handle while the target was accurately enough aligned with the dock. Time required to reacquire handles after errors is included in this measure. An ANOVA suggests that dragging time was significantly shorter in the direct $(M=3500, S D=1619)$ than in the indirect condition $(M=4315, S D=2358), F_{1,21}=31.39, p<0.001$.

Both, width and distance, have main effects on dragging time, $F_{2,42}=6.44$, $p=0.004$ and $F_{2,42}=43.23, p<0.001$ for width and distance respectively. No significant interaction between any factors was found.

Dragging Errors. A dragging error occurred when either handle was left while dragging. Multiple dragging errors could occur within a single trial. An ANOVA indicates a significant main effect of condition on dragging errors, $F_{1,21}=5.72, p=0.026$. Participants completed the dragging subtask with fewer errors in the direct $(M=0.36$, $S D=1.3)$ than in the indirect condition $(M=0.68, S D=2.64)$. No further significant effects were found.

Handle Selection Delay. Furthermore, we measured the delay in milliseconds between the initial selection of the first and second handle. An ANOVA revealed that this delay was significantly lower $\left(F_{1,21}=36.43, p<0.001\right)$ in the direct $(M=133$, $S D=317)$ than indirect $(M=525, S D=595)$ condition.

Both, width and distance, have main effects on handle selection delay, $F_{2,42}=71.47, p<0.001$ and $F_{2,42}=7.7, p=0.001$ for width and distance respectively. Additionally, the condition $\times$ width interaction is significant, $F_{1.6,34.75}=11.51$, $p<0.001$ (Huynh-Feldt). No further interactions were found.

\subsection{Observations}

The following descriptions are based on observations made during the experiment and on a post-hoc analysis of the recorded video. In general, the task was easily understood by the participants, independent of which condition was tested first.

However, a noticeable difference regarding the way users approached the surface with their arms and hands could be observed. While many participants relaxed their hands by slightly changing the hands' postures or moving them around the wrist in between trials when interacting directly on the table, their hands remained in a rather static posture, mostly hovering over the surface and already focusing on moving to the home area again when interacting with the screen. We also observed that users often maintained a more tense hand posture in the indirect than in the direct condition. In this context, several users were intuitively relaxing and shaking their hands in between blocks in the indirect condition.

In addition, we could observe that participants were more likely to break down the pointing movements in the indirect condition. First, they aligned their fingers with the target to point at. Second, they moved their fingers down to touch the surface. In 
the direct condition, the pointing task resembled more a fluid single movement. Moreover, it was obvious that many participants followed a sequential strategy to select the two handles of a target in the indirect condition. Only after they positioned their finger over one handle and then put it down to make the selection, they started to adjust the position of their other hand's finger.

We observed that several users tried to apply a different strategy in the pointing task when interacting with the screen. They left their fingers on the surface while sliding towards the target, then lift the fingers up and put them down again to select the target. In these cases, users were asked to perform the pointing as originally instructed.

A potential problem which we noted in this user study concerns long finger nails. They prevent users from touching the table in a steep angle which causes more unintended touches. Moreover, they make the fingers appear longer in the hand contour representation which results in a mismatch between the displayed fingertip and the actual point of touch.

\subsection{User Feedback}

Quantitative Feedback. Participants consistently rated the direct condition better in both, the satisfaction questionnaire and the task load index. Wilcoxon Signed-Rank Tests indicate that these differences are significant for each rating.

Results show that 3 out of $22(13.64 \%)$ participants preferred the indirect condition. Moreover, three (different) participants stated that they were faster in the indirect condition; two of them also felt they made fewer errors in it. In addition, one of those who preferred the indirect condition also stated he made fewer errors in it. However, none of these ratings regarding time and error rate are reflected in the collected data.

Qualitative Feedback. In addition to the previous ratings, we asked participants to comment on positive as well as negative aspects of the tested conditions during and in the end of the experiment. Ten participants perceived the direct condition to be easier than the indirect condition. Five participants noted that more mental effort was required to complete the tasks in the indirect condition; they felt they had to concentrate and think more or were more tired afterwards. Twelve of the participating users stated that it was difficult to coordinate their hands with the respective contours displayed on the screen. In addition, two of them reported on having problems with distinguishing their fingers on the screen, hence they only used their index fingers in a pointing posture.

In the direct condition, two participants said they had experienced problems with occlusion due to their arms or hands covering parts of the table; three participants felt they had a better overview or visibility with the screen. We received five comments indicating that the indirect condition is more comfortable mainly due to problems that occur in the direct condition when looking down at the table for a prolonged period of time. However, further seven participants perceived the direct condition as more comfortable to work with; three of them noted that this is due to a higher amount of physical stress for the fingers in the indirect condition.

Further comments reached from describing the indirect setup as a fun system with an appealing idea that one user even could imagine to be working with comfortably in a job over the day, to statements about how nerve breaking this kind of interaction 
was perceived. One user noted that she hated the indirect condition while another saw it as a training tool for multitasking abilities.

\section{Discussion}

In general, completion times were shorter and error rates lower in the direct condition throughout all results. This difference in speed and accuracy was also perceived by most participants when asked for a comparative assessment of the two conditions. In addition, results of task load index show that participants rated their performance better in the direct than in the indirect condition. Furthermore, agreement with the two statements of the usability satisfaction questionnaire which are concerned with speed and efficiency also underlinesS these results.

One possible explanation for this measured and perceived performance decrease can be found in the additional cognitive load present in the indirect condition. Not only the task load evaluation revealed a higher demand for mental and perceptual activity in the indirect condition, also the qualitative feedback we received suggested that more concentration was required. In addition, the input hardware was identical in both conditions which suggests that observed differences are due to an added complexity of coordination. In summary, interacting indirectly with the system was more challenging for participants and resulted in a significantly higher frustration level.

\subsection{Differences in Pointing and Dragging}

Times. In accordance with findings of related studies [9, 18], dragging was slower than pointing, independent of the condition. It is noteworthy that the overall trial time was decreased by the same factor in our study as in the one by Forlines et al. [9], although different input techniques were used in the indirect condition. Additionally, it is important to acknowledge that the dragging was more demanding than the pointing task by design since it included positioning and scaling. However, while it took participants on average $2390 \mathrm{~ms}(55 \%)$ longer to complete a trial in the indirect condition, the already shorter pointing time contributed $1575 \mathrm{~ms}(66 \%)$ to this total time difference between direct and indirect interaction; only $815 \mathrm{~ms}$ were due to differences in dragging performance. That is, it took participants about $200 \%$ longer to complete a pointing task in the indirect condition, while we only observed a time decrease of $23 \%$ for the dragging task. These results suggest that differences in the conditions had more impact on completion time for pointing than dragging.

Moreover, significant interactions between condition and width as well as distance were found for pointing time. Contrasts on these interaction terms reveal that the smaller the target is, the larger is the pointing time difference between direct and indirect condition. On the same lines, larger distances have a higher impact on pointing time in the indirect condition. That is, smaller targets respectively larger distances seem to render the pointing task unequally more time consuming in the indirect compared to the direct condition. In contrast to pointing time, there is no indication that width or distance had a significant influence on the difference in dragging time between conditions, suggesting that there is less complexity added in the indirect condition compared to pointing. 
While our experiment was designed to include error trials it is still insightful to look at times without errors. A brief analysis of only those pointing tasks without errors reveals completion times which are around $11 \%$ lower in both conditions; indirect pointing is still about $200 \%$ slower. However, an analog analysis for dragging times shows that the speed advantage of direct over indirect interaction for dragging is further decreased. In trials without errors we only observed a time decrease of $13 \%$ for dragging between the direct and indirect condition, also indicating a smaller discrepancy in complexity between direct and indirect interaction.

Errors. Since a trial was not interrupted on errors but continued until successfully completed, it is no surprise to find a similar tendency of differences between interaction conditions as seen for completion times in the respective error rates, too. While participants were more than three times as likely to make a pointing error in the indirect than direct condition, a dragging error was only less than twice as likely made.

Yet it is important to account for the relatively high dragging error rates observed in our study compared to values found in literature for a similar task [9]. First, it was sufficient to position the target over the dock in the said study; matching was performed automatically. Our experiment required participants to actively release the handles, though, resulting in a more complex and error prone task. Second, we used a vision-based input technology which is more sensitive to touches and capable of detecting non-finger contacts, too. In specific, we observed that lifting the finger off the surface sometimes caused the selected target to move as described by Sears et al. [23]. Therefore, it was not properly aligned with the dock anymore which was counted as dragging error. Third, our prototype system sometimes lost momentarily track of fingers while dragging which is indicated by frequent handle reselection events within milliseconds. This happened in both conditions at about the same rate. It was post-hoc not possible to distinguish these errors from valid user activities.

Furthermore, it is interesting to note that no significant interactions were found between width or distance and condition for the pointing error rate. In the light of the findings about pointing time, this indicates that smaller targets or larger distances did not have a pronounced effect on pointing error rate in the indirect compared to the direct condition. In short, it appears that the time spent for aiming paid off.

\subsection{Peculiarities of Indirect Interaction}

In the following, we discuss observed behavior which gave indication to higher cognitive load in the indirect condition and helps to explain the varying performance differences between direct and indirect condition and pointing and dragging tasks. A first indicator for an increased mental demand is the less parallel approach of handle selection in the indirect condition. Analyzing the time which passed between selecting the first and second handle of a target shows that participants were about four times quicker to select the second handle in the direct condition, also contributing to the total shorter pointing time.

As described before, we observed that participants tended to split the pointing task into two separate movements. Only after positioning the fingers over the target they would move them down to touch the surface. A possible explanation for this behavior can be found in the increased difficulty of estimating the distance between hand and table surface when looking at the screen; the user interface did not provide 
three-dimensional hints. Therefore, it is arguably more difficult to perform a fluid movement consisting of a combined horizontal and vertical component to select a target in the indirect condition. Participants attempting to perform such a combined movement often touched the surface too early and slid into the target instead of pointing at it which accounted for a pointing error. To sum up, the missing perception of the hands' three-dimensional location above the surface seems to substantially add to the coordination load in the indirect condition. Clearly, no estimation of distance between hands and surface is required while dragging. In addition, observations of users who tried to apply a sliding and a "lift-and-tap" technique [17] instead of a pointing movement suggest that dragging was perceived to be more intuitive in the indirect condition. However, realizing selection by dragging raises challenges concerning the differentiation of input states as discussed by Buxton et al. [4]. Wilson et al. [25] present a pinching gestures which could serve as trigger for a state transition. On the same lines, Benko et al. [3] implemented SimPress to distinguish between mere touches and clicks.

In general, we could observe a more restricted usage of the indirect interaction technique with regard to hand postures and movements. Participants seemed to put more emphasis in maintaining certain hand postures over the trials in the indirect condition. A possible explanation for this behavior is the added complexity due to the mapping between the hand contours and the actual hands. This mapping can arguably be facilitated by having a clear and constant point of reference, such as the index finger in a pointing gesture.

\section{Conclusions}

Our results indicate that a simple replication and transfer of common interaction techniques from a direct multi-touch to an indirect multi-touch surface comes along with a substantial decrease in performance. Nevertheless, it is important to acknowledge that even unexperienced users understood the underlying principles of the studied indirect interaction modality instantly. In addition, our results indicate that performance loss between direct and indirect is mostly due to the requirement of blindly keeping arms and hands at distance to the input device. Allowing the user to stay in contact with the surface has been shown to decrease performance differences between direct and indirect interaction considerably. These findings motivate the design of interaction techniques that allow the user to keep permanent contact with the surface. The presented results provide a basic understanding of factors relevant to the design of indirect multi-touch systems and suggest that a significant potential for improving indirect multi-touch interaction exists. Further investigations with respect to the quality of indirect multi-touch interaction is clearly needed to gain a better understanding of costs and benefits of these techniques.

\section{References}

1. Balakrishnan, R.: The Role of Kinesthetic Reference Frames in Two-Handed Input Performance. In: Proc. UIST, pp. 171-178 (1999)

2. Barnert, W.C.: A Comparison of One-Handed and Two-Handed Direct and Indirect Computer Interaction. Technical report, Tufts University (2005) 
3. Benko, H., Wilson, A.D., Baudisch, P.: Precise Selection Techniques for Multi-Touch Screens. In: Proc. CHI, pp. 1263-1272 (2006)

4. Buxton, W.: A Three-State Model of Graphical Input. In: Proc. INTERACT, pp. 449-456 (1990)

5. Buxton, W., Myers, B.: A Study in Two-Handed Input. In: Proc. CHI, pp. 321-326 (1986)

6. Cao, X., Wilson, A.D., Balakrishnan, R., Hinckley, K., Hudson, S.E.: ShapeTouch: Leveraging Contact Shape on Interactive Surfaces. In: Proc. TABLETOP (2008)

7. Dietz, P., Leigh, D.: DiamondTouch: A Multi-User Touch Technology. In: Proc. UIST, pp. 219-226 (2001)

8. Forlines, C., Balakrishnan, R.: Evaluating Tactile Feedback and Direct vs. Indirect Stylus Input in Pointing and Crossing Selection Tasks. In: Proc. CHI, pp. 1563-1572 (2008)

9. Forlines, C., Wigdor, D., Shen, C., Balakrishnan, R.: Direct-Touch vs. Mouse Input for Tabletop Displays. In: Proc. CHI, pp. 647-656 (2007)

10. Han, J.Y.: Low-Cost Multi-Touch Sensing Through Frustrated Total Internal Reflection. In: Proc. UIST, pp. 115-118 (2005)

11. Hart, S.G., Stavelan, L.E.: Development of NASA-TLX (Task Load Index): Results of Empirical and Theoretical Research. Human Mental Workload (1988)

12. Krueger, M.W., Gionfriddo, T., Hinrichsen, K.: VIDEOPLACE - An Artificial Reality. SIGCHI Bull. 16(4), 35-40 (1985)

13. Kurtenbach, G., Fitzmaurice, G., Baudel, T., Buxton, W.: The Design of a GUI Paradigm Based on Tablets, Two-Hands, and Transparency. In: Proc. CHI, pp. 35-42 (1997)

14. Lee, S.K., Buxton, W., Smith, K.C.: A Multi-Touch Three Dimensional Touch- Sensitive Tablet. SIGCHI Bull. 16(4), 21-25 (1985)

15. Leganchuk, A., Zhai, S., Buxton, W.: Manual and Cognitive Benefits of Two-Handed Input: An Experimental Study. ACM Trans. Comput.-Hum. Interact. 5(4), 326-359 (1998)

16. Lewis, J.R.: IBM Computer Usability Satisfaction Questionnaires: Psychometric Evaluation and Instructions for Use. Int. J. Hum.-Comput. Interact. 7(1), 57-78 (1995)

17. MacKenzie, I.S., Oniszczak, A.: A Comparison of Three Selection Techniques for Touchpads. In: Proc. CHI, pp. 336-343 (1998)

18. MacKenzie, I.S., Sellen, A., Buxton, W.: A Comparison of Input Devices in Elemental Pointing and Dragging Tasks. In: Proc. CHI, pp. 161-166 (1991)

19. Malik, S., Ranjan, A., Balakrishnan, R.: Interacting with Large Displays from a Distance with Vision-Tracked Multi-Finger Gestural Input. In: Proc. UIST, pp. 43-52 (2005)

20. Meyer, S., Cohen, O., Nilsen, E.: Device Comparisons for Goal-Directed Drawing Tasks. In: Proc. CHI, pp. 251-252 (1994)

21. Moscovich, T., Hughes, J.F.: Indirect Mappings of Multi-Touch Input Using One and Two Hands. In: Proc. CHI, pp. 1275-1284 (2008)

22. Scott, S.S., Carpendale, S.: Guest Editors' Introduction: Interacting with Digital Tabletops. IEEE Computer Graphics and Applications 26(5), 24-27 (2006)

23. Sears, A., Shneiderman, B.: High Precision Touchscreens: Design Strategies and Comparisons with a Mouse. IJMMS 34, 593-613 (1991)

24. Wigdor, D., Forlines, C., Baudisch, P., Barnwell, J., Shen, C.: Lucid Touch: A SeeThrough Mobile Device. In: Proc. UIST, pp. 269-278 (2007)

25. Wilson, A.D.: Robust Computer Vision-Based Detection of Pinching for One and TwoHanded Gesture Input. In: Proc. UIST, pp. 255-258 (2006)

26. Wu, M., Balakrishnan, R.: Multi-Finger and Whole Hand Gestural Interaction Techniques for Multi-User Tabletop Displays. In: Proc. UIST, pp. 193-202 (2003) 